\title{
Iris metastasis from esophageal squamous cell carcinoma: A case report
}

\author{
DONGLAI LV ${ }^{1}$, ZONGTAO HU ${ }^{1}$, CHONG WANG ${ }^{1}$, SHILE GAO $^{1}$ and JUN XU ${ }^{2}$ \\ Departments of ${ }^{1}$ Clinical Oncology and ${ }^{2}$ Pathology, The 105 Hospital of The People's Liberation Army, \\ Hefei, Anhui 230031, P.R. China
}

Received August 25, 2014; Accepted May 12, 2015

DOI: 10.3892/ol.2015.3255

\begin{abstract}
Carcinoma metastatic to the eye is a rare condition, typically associated with a poor prognosis. Breast and lung cancers are the most common sources of intraocular metastases, and the majority of metastatic lesions involve the posterior uvea, with $<8 \%$ of reported cases arising in the iris. Intraocular metastasis as the presenting form of esophageal carcinoma is highly uncommon. In the present report, a rare case of metastatic iris tumor resulting from esophageal squamous cell carcinoma is discussed. A 64-year-old patient presented with a progressively distending pain in the right eye, with associated blurred vision. Local and systemic evaluation was performed, followed by treatment. Multiple examinations identified a neoplasm in the right iris and postoperative pathology revealed that the iris lesion was a metastasis of esophageal squamous cell cancer origin. The patient was treated with adjuvant radiation. To the best of our knowledge, this was only the second reported case of esophageal squamous cell carcinoma metastasizing to the iris.
\end{abstract}

\section{Introduction}

Esophageal cancer is an aggressive disease that typically metastasizes to the lymph nodes, lungs and liver (1), while its metastasis to the eyes is extremely rare. Breast and lung cancers are the source of the majority of malignant intraocular metastatic tumors (2). To date, several cases of esophageal carcinoma with intraocular metastasis have been reported (3-5), although these were all adenocarcinomas and spread to the choroid but not the iris. Previous studies indicated that while metastatic lesions mainly invade the posterior uvea, and in particular the choroid, a small portion may arise in the iris, ciliary body or retina $(6,7)$. This is often

Correspondence to: Dr Donglai Lv, Department of Clinical Oncology, The 105 Hospital of The People's Liberation Army, 424 Changjiang West Road, Hefei, Anhui 230031, P.R. China

E-mail: lvxunhuan@163.com

Key words: esophageal cancer, iris metastasis, squamous cell carcinoma considered to be due to the particular anatomical structure: The posterior aspect of the uvea (choroid) has very rich blood supply but the iris has few arteries, therefore the tumor cells easily arrive and seed into the choroid through short posterior ciliary artery. The choroidal blood supply is abundant, and characterized by large lumen with a small volume of blood imported and exported; metastatic cancer cells are easily able to seed here (8). In the present report, a rare case of a patient presenting with right eye lesion due to metastasis of esophageal squamous cell carcinoma is discussed. Furthermore, the present report identifies the potential diagnoses and treatment of this type of tumor. Written informed consent was obtained from the patient.

\section{Case report}

A 64-year-old male, with a history of esophageal squamous cell cancer diagnosed 13 months previously, which was treated by total esophagectomy (stage IIa, pT3N0M0) and postoperative radiotherapy and chemotherapy, sought treatment for a two-week history of a progressively distending pain in the right eye with associated blurred vision.

The patient's original computed tomography (CT) (Fig. 1A) and postoperative pathology results (Fig. 1B) revealed a lower-differentiation squamous cell carcinoma. The specimen stained positive for immunohistochemical (IHC) markers for P63 (Fig. 1C). Additional medical history included hypertension and gastric stromal tumor (low risk). However, the patient had no previously known ophthalmic diseases.

Ophthalmic examination was subsequently conducted. Best-corrected visual acuity was 20/400 in the right eye and $20 / 25$ in the left eye. Intraocular pressures, extraocular motility, confrontation fields, external exam and slit-lamp examination of the left eye were all normal. The right eye exhibited blepharospasm, conjunctival hyperemia, corneal slight edema and high intraocular pressure $(25 \mathrm{mmHg})$. Slit-lamp examination of the right eye identified a gray, irregular and vascularized neoplasm on the temporal side of the iris. CT examination (Fig. 2A) and ophthalmic ultrasound verified the neoplasm in the right eye. The patient underwent ophthalmectomy of the right side. Postoperative pathology revealed the tumor to be $0.7 \times 0.5 \times 0.3 \mathrm{~cm}$ in size and confirmed the diagnosis of a metastatic iris neoplasm 

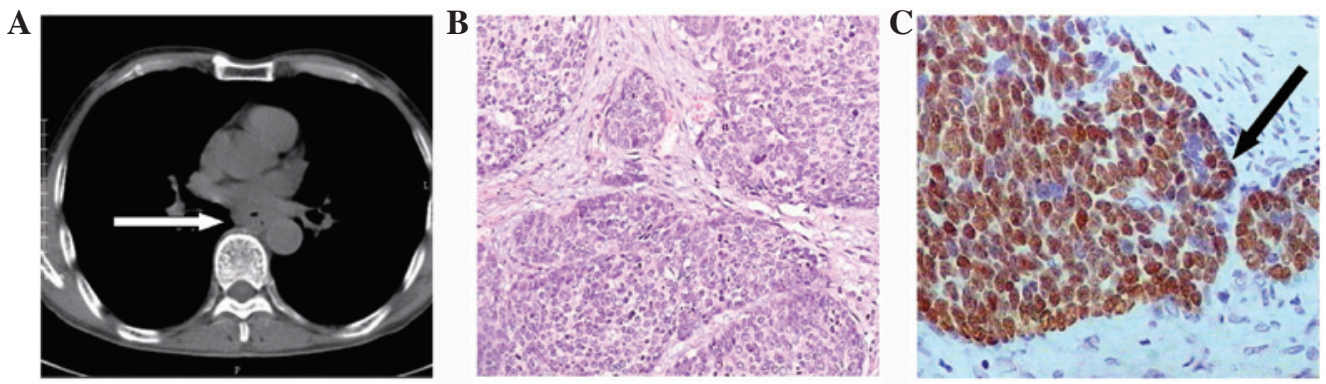

Figure 1. CT and histopathological analysis of the esophageal primary cancer. (A) CT scan identified neoplastic tissue (arrow) in the lower esophagus. (B) Poorly differentiated esophageal squamous cell carcinoma identified by postoperative pathological analysis (hematoxylin and eosin staining; magnification, x100). (C) Immunohistochemical staining for P63 within the areas of carcinoma nests (arrows) from the primary specimen (magnification, x200). CT, computed tomography.
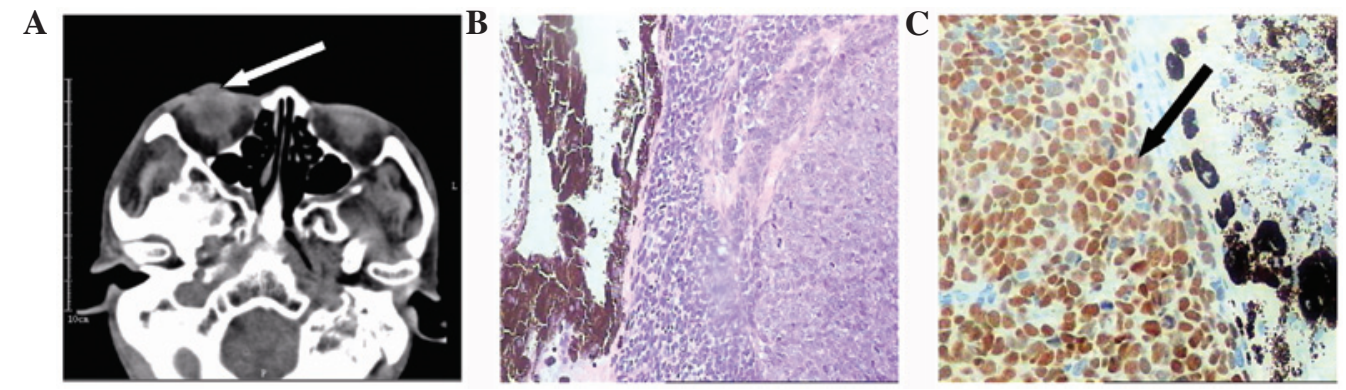

Figure 2. CT and histopathological analysis of the iris neoplasm. (A) CT scan identified neoplastic tissue (arrow) in the right eye. (B) Carcinoma nests invaded the iris and a worse differentiation degree was observed in the postoperative pathology of the iris neoplasm than the primary tumor (hematoxylin and eosin staining; magnification, x100). (C) Immunohistochemical staining for P63 (arrows) from the metastatic specimen were consistent with primary esophageal carcinoma (magnification, x200).CT, computed tomography.

of esophageal squamous cell cancer origin (Fig. 2B and C). Subsequently, adjuvant radiation with a total dose of $40 \mathrm{~Gy}$ was administered, but the patient refused postoperative chemotherapy.

\section{Discussion}

Ocular metastases of malignant gastrointestinal tumors are rare and have a poor prognosis of just 13 months median survival following diagnosis. Furthermore, iris metastases comprise $<8 \%$ of all ocular metastases reported within the literature (9). The most common primary sites of ocular metastasis are breast cancer in females and lung cancer in males. In addition, sarcomas and melanomas may also metastasize to the eye (10). For esophageal carcinoma, only two cases of iris metastases have been reported in the last 20 years, one of which was adenocarcinoma (11) and the other was moderately differentiated squamous cell carcinoma (12). To the best of our knowledge, the present report was therefore only the second reported case of esophageal squamous cell carcinoma metastasis to the iris.

Iris metastasis has a variety of clinical manifestations, including decreased vision, ophthalmodynia, diplopia, proptosis and periorbital swelling (13). The present patient sought medical advice once the blurred vision had almost caused blindness, and a cataract was suspected.

This rare case highlights a number of important considerations. In clinical work, particular attention should be paid to the ocular lesions of patients with a history of cancer.
Furthermore, it is necessary to perform comprehensive and serious systemic and specialist examinations in order to avoid misdiagnosis.

The diagnosis of ocular metastasis is often difficult. Ophthalmic examination with slit-lamp biomicroscopy and indirect ophthalmoscopy are important diagnostic tools for use upon initial examination. Furthermore, ocular ultrasound, $\mathrm{CT}$, magnetic resonance imaging and positron emission tomography (PET)/CT examination are used as powerful diagnostic tools. In particular, PET/CT scans may aid the identification of benign or malignant lesions and indicate the potential primary site. However a clear history of a systemic malignancy is still the most significant factor. Biopsy and immunohistochemical staining may be used for the final diagnosis, to characterize the neoplasm and determine the primary site, particularly in certain rare, poorly-differentiated cases (14).

To date, the treatments for ocular metastasis available include radiation, laser, chemotherapy, anti-vascular endothelial growth factor and eye enucleation (10). More than half of patients exhibit another metastatsis at the time the ocular lesion is detected (2), therefore treatment is typically non-curative. Taking into account the severe eyeball pain of the patient and the inability to restore vision, eyeball enucleation and adjuvant radiation was selected in the present case.

A notable contrast was also identified: The pathological results demonstrated that the cellular differentiation degree of the ocular metastatic lesion was worse than that of the primary tumor site. It was suggested that this may be attributed to the 
metastatic lesion originating from residual cancer stem cells following multiple treatments, which have a worse phenotype and exhibit drug-resistance subsequent to relapse. This highlighted the importance of targeting cancer stem cell therapy in the future.

\section{References}

1. Kato $\mathrm{H}$ and Nakajima M: Treatments for esophageal cancer: A review. Gen Thorac Cardiovasc Surg 61: 330-335, 2013.

2. Shields CL, Shields JA, Gross NE, Schwartz GP and Lally SE: Survey of 520 eyes with uveal metastases. Ophthalmology 104: 1265-1276, 1997.

3. Knezevic J, Radovanovic N, Simic A, Bobić Radovanović A, Latković Z, Micev M and Pesko P: Isolated choroidal metastasis from primary adenocarcinoma of the distal esophagus: A case report. Dis Esophagus 16: 41-43, 2003.

4. Buskens CJ, Tan HS, Hulscher JB, de Smet MD and van Lanschot JJ: Adenocarcinoma of the esophagus with choroidal metastasis. Dis Esophagus 14: 70-72, 2001.

5. Parikh HK, Deshpande RK, Swaroop DV and Desai PB: Choroidal metastasis from primary adenocarcinoma of the esophagus - a case report. Indian J Cancer 29: 210-214, 1992.
6. d'Abbadie I, Arriagada R, Spielmann M and Lê MG: Choroid metastases: Clinical features and treatments in 123 patients. Cancer 98: 1232-1238, 2003.

7. Kim CY, Ha CW and Lee SC: Vitreous and retinal metastasis from gastric cancer. Eur J Ophthalmol 20: 615-617, 2010.

8. Albert DM, Zimmermann AW Jr and Zeidman I: Tumor metastasis to the eye. 3. The fate of circulating tumor cells to the eye. Am J Ophthalmol 63: 733-738, 1967.

9. Shields JA, Shields CL, Kiratli H and de Potter P: Metastatic tumors to the iris in 40 patients. Am J Ophthalmol 119: 422-430, 1995.

10. Giuliari GP and Sadaka A: Uveal metastatic disease: Current and new treatment options (review). Oncol Rep 27: 603-607, 2012.

11. Lee WB, Sy HM, Filip DJ and Grossniklaus HE: Metastatic esophageal adenocarcinoma presenting in the iris. Am J Ophthalmol 144: 477-479, 2007.

12. Ichiki Y, Morita M, Yano K, Sugio K, Yasumoto K and Hirose N: Iris metastasis of esophageal cancer. Ann Thorac Surg 79: 1782-1784, 2005

13. Freedman MI and Folk JC: Metastatic tumors to the eye and orbit. Patient survival and clinical characteristics. Arch Ophthalmol 105: 1215-1219, 1987.

14. Kanthan GL, Jayamohan J, Yip D and Conway RM: Management of metastatic carcinoma of the uveal tract: An evidence-based analysis. Clin Experiment Ophthalmol 35: 553-565, 2007. 